\title{
Tricho-Rhino-Phalangeales Syndrom
}

\author{
Tricho-Rhino-Phalangeal Syndrome
}

Autor

Institut
W.-I. Worret

Klinik und Poliklinik für Allergologie und Dermatologie am Biederstein der Technischen Universität München (Dir.: Univ.-Prof. Dr. med. Dr. phil. J. Ring)
Bibliografie

DOI http://dx.doi.org/ $10.1055 / \mathrm{s}-0029-1215005$

Online-Publikation: 25. 8. 2009

Akt Dermatol 2010; 36:

91-93 @ Georg Thieme

Verlag KG Stuttgart · New York ISSN 0340-2541

Korrespondenzadresse

Prof. Dr. med.

\section{Wolf-Ingo Worret}

Klinik und Poliklinik für Dermatologie am Biederstein des Klinikums rechts der Isar der TU München

Biedersteiner Str. 29

80802 München

wolf-ingo.worret@|rz.tum.de

\section{Zusammenfassung \\ $\nabla$}

Das Tricho-Rhino-Phalangeale Syndrom ist ein sehr seltenes Krankheitsbild, welches aber klinisch meist recht einfach zu diagnostizieren ist. In dieser Kasuistik wird eine neue Patientin beschrieben und die typischen Symptome herausgearbeitet.

\section{Einleitung}

$\nabla$

Mehr als 150 distinkte Syndrome wurden beschrieben, bei welchen es zu Entwicklungsstörungen der Haare kommt, und es ist demnach sehr schwierig, alle diese Fehlbildungen zu überblicken. Insbesondere besteht keine allgemein anerkannte Klassifikation. Man kann die Syndrome klassifizieren in unterschiedliche Erbgänge [2], genetische Aberrationen [3] oder aufgrund der klinischen Bilder. Letztere Klassifizierung ist gerade bei den angeborenen Hypotrichosen (HT) einigermaßen praktikabel [5]. So kann man unterteilen in HT ohne assoziierte Defekte (z.B. MarieUnna HT), HT mit assoziierten Defekten (z.B. mit mentalen Retardierungen, Skelettanomalien oder Ichthyosen) und die große Gruppe der HT verbunden mit einem ektodermalen Dysplasie-Syndrom (z.B. Abnormalitäten der Zähne, Nägel oder Schweißdrüsen).

\section{Anamnese \\ $\nabla$}

17-jährige Patientin, die seit dem 3. Lebensjahr an einer Haarwachstumsstörung leidet. Die Patientin ist Einzelkind und in der Familie sowie in der Verwandtschaft sind keine speziellen Hautveränderungen oder Krankheiten bekannt.

\section{Befunde \\ $\nabla$}

Klinischer Befund

Betroffen ist das Capillitium. Man sieht spärliche, feine, pigmentierte Haare und unregelmäßige kahle Stellen ( $\bullet$ Abb. 1). Ein Effluvium besteht jedoch nicht. Die Augenbrauen sind schwach ausgebildet. Weiter fallen eine birnenförmige Nase, ein verlängertes Philtrum, etwas abstehende Ohren und verkürzte Finger sowie zarte und brüchige Fingernägel auf; Fehlstellungen der Frontalzähne. Die Weisheitszähne fehlen. Die Patientin ist etwas klein, aber psychisch altersentsprechend entwickelt $($ Abb. 2).

\section{Laborbefunde}

Die Routinelaborparameter und der Hormonstatus sind altersentsprechend im Normbereich.

\section{Radiologische Befunde}

Die ap-Aufnahmen zeigten eine deutliche Verkürzung der Mittelphalanx und Endphalanx D I bds. sowie eine deutliche Verkürzung der Mittelphalanx D II und D V bds. Keine Epiphysenbereiche mehr abgrenzbar. Regelrechte Darstellung des Handwurzelskeletts sowie des Radius und der Ulna. Keine Arthroseanzeichen.

Die Randkanten der Mittelphalanx D II und D IV zeigten sich basalseitig leicht ausgezogen, gleichermaßen wie das Daumengrundgelenk und das Endglied beidseits. Zusammenfassend sind die Veränderungen des Endgliedes D I mit der deutlichen Verkürzung sowie der Mittelphalanx D II und D V typisch für das Vorliegen eines Tricho-Rhino-Phalangealen Syndroms ( Abb. 3 und 4).

\section{Trichogramm}

Bei der Untersuchung zeigten sich 23\% Anagenhaare ohne Wurzelscheiden, 38\% abgebrochene Haare, 37\% Telogenhaare und 2\% dystrophische Haare. 

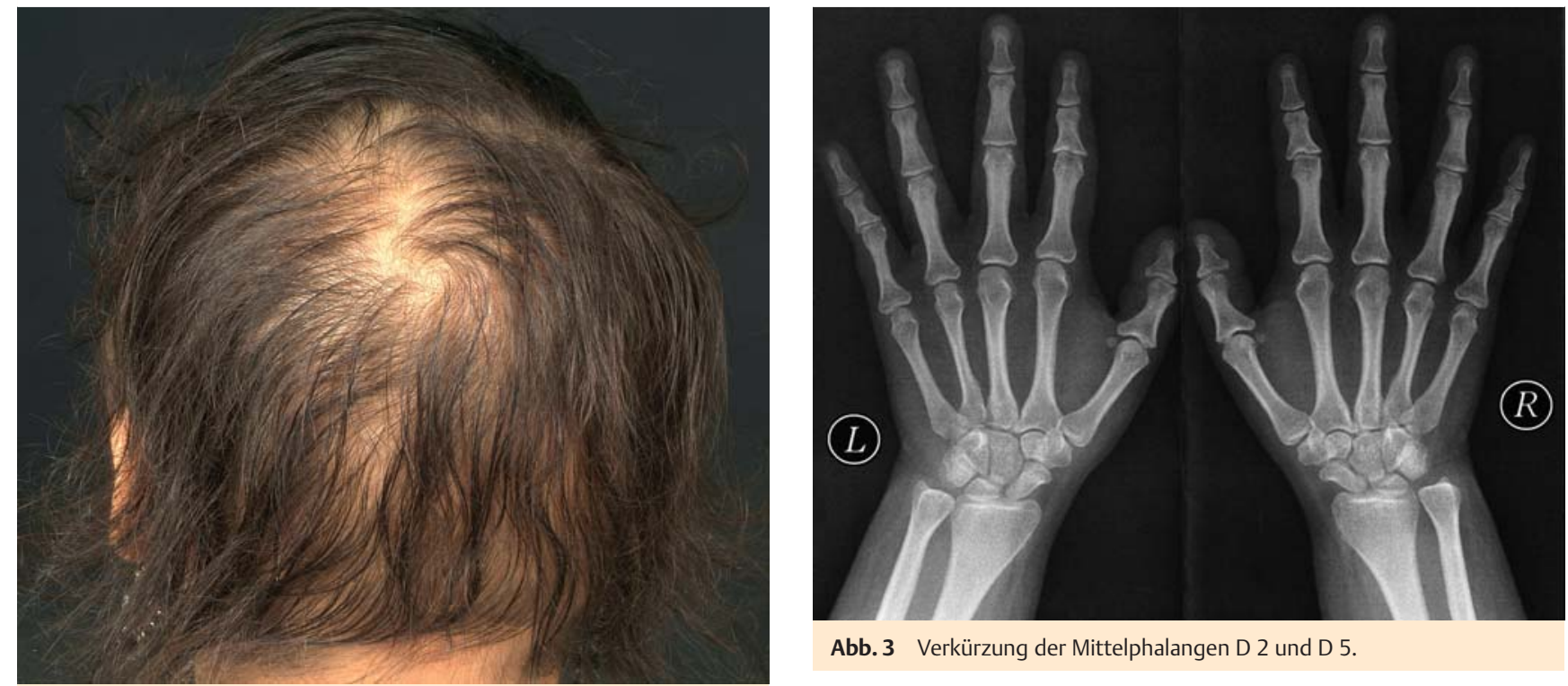

Abb. 3 Verkürzung der Mittelphalangen D 2 und D 5.

Abb. 1 Hypotrichose am Hinterkopf.
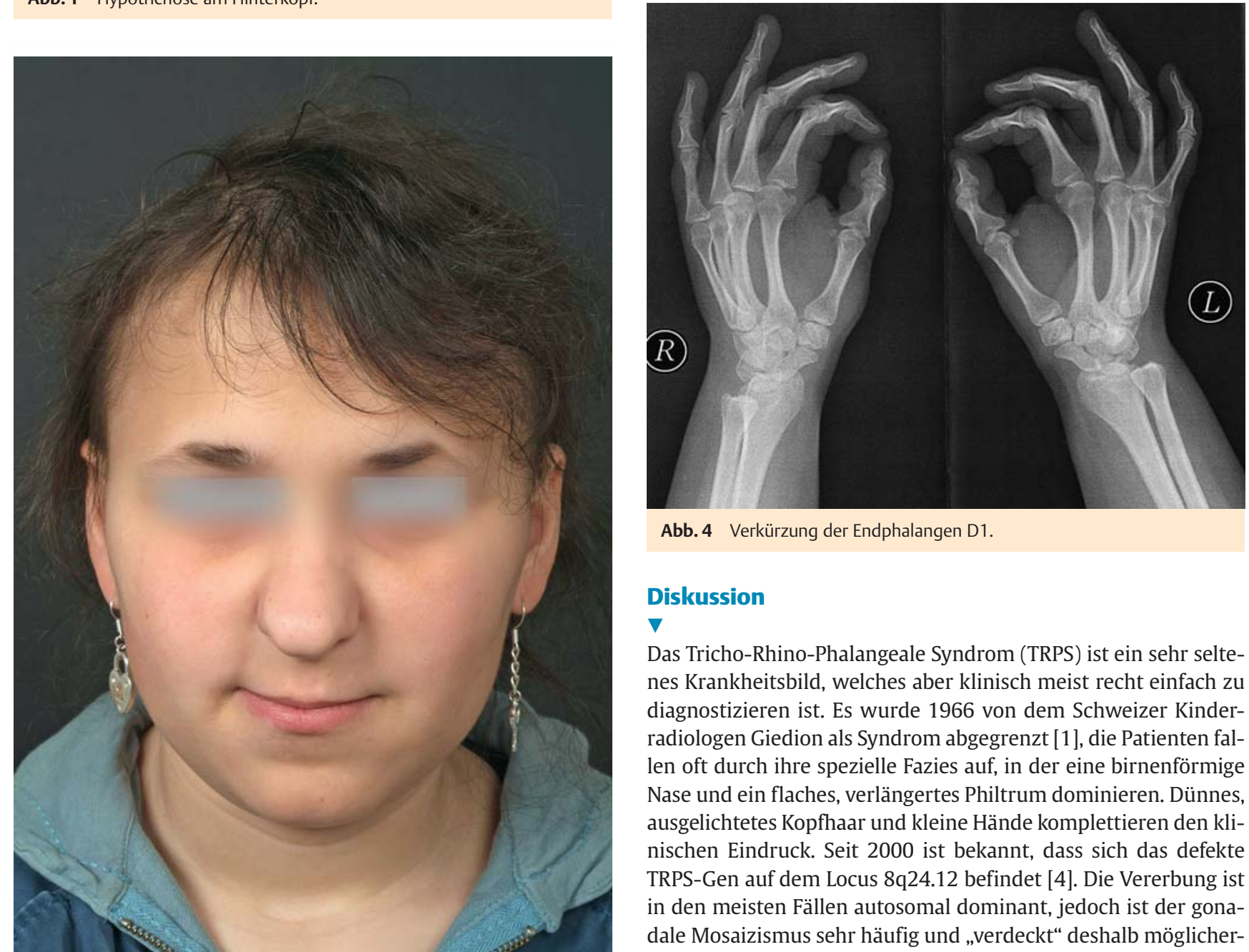

Abb.4 Verkürzung der Endphalangen D1.

\section{Diskussion}

$\nabla$

Das Tricho-Rhino-Phalangeale Syndrom (TRPS) ist ein sehr seltenes Krankheitsbild, welches aber klinisch meist recht einfach zu diagnostizieren ist. Es wurde 1966 von dem Schweizer Kinderradiologen Giedion als Syndrom abgegrenzt [1], die Patienten fallen oft durch ihre spezielle Fazies auf, in der eine birnenförmige Nase und ein flaches, verlängertes Philtrum dominieren. Dünnes, ausgelichtetes Kopfhaar und kleine Hände komplettieren den klinischen Eindruck. Seit 2000 ist bekannt, dass sich das defekte TRPS-Gen auf dem Locus 8q24.12 befindet [4]. Die Vererbung ist in den meisten Fällen autosomal dominant, jedoch ist der gonadale Mosaizismus sehr häufig und „verdeckt“ deshalb möglicherweise rezessive Formen. Bis heute wurden 3 Typen herausgearbeitet [3]. Bei Typ I und III besteht eine Haploinsuffizienz eines spezifischen Zinkfinger-Proteins, welches einen möglichen Transkriptionsfaktor darstellt. Klinisch kann man diese Typen unterscheiden, indem beim TRPS I kegelförmige Epiphysen der Phalangen gefunden werden, evtl. auch Hüftmalformationen, während beim TRSP III- oder auch Sugio-Kajii-Syndrom eine starke Verkürzung der Metacarpalphalangen und/oder der Metatar- 
salphalangen vorliegt [6]. Im Gegensatz dazu findet man bei TRPS II, auch Langer-Giedion-Syndrom, mentale Defizite und Exostosen.

Bei der hier vorgestellten Patientin handelt es sich am wahrscheinlichsten um ein TRPS I, da die Metacarpale vornehmlich betroffen sind, die Mittelhandknochen nicht verändert sind und eine altersentsprechende Intelligenz vorliegt. Die typische birnenförmige Nase, verbunden mit dem schütteren Kopfhaar, was ja bei allen Patienten der TRPS-Gruppe gefunden wird, wies den Weg zur Gruppe der Tricho-Rhino-Palangealen Syndrome, die Röntgenaufnahme der Hände führte dann zur Diagnose. Eine Therapie der Hypotrichose ist nicht möglich.

\section{Abstract}

\section{Tricho-Rhino-Phalangeal Syndrome}

The Tricho-rhino-phalangeal syndrome is a very rare disease pattern, but is clinically frequently diagnosed in a quite simple manner. In this report a new female patient is described and the typical symptoms are elaborated.

\section{Literatur}

1 Giedion A. Das Tricho-rhino-phalangeal Syndrom. Helv Paediat Acta 1966; 21: $475-482$

2 Happle R. Genetic defects involving the hair. In: Orfanos $\mathrm{CD}$, Happle $\mathrm{R}$ (eds). Hair and Hair Diseases. Heidelberg: Springer, 1990: 325-362

3 McKusick VA. Mendelian Inheritance in Man; A Catalog of Human Genes and Genetic Disorders. Baltimore, Maryland: The Johns Hopkins University Press, 1998

4 Momeni P, Glockner G, Schmidt O et al. Mutations in a new gene, encoding a zinc-finger protein, cause tricho-rhino-phalangeal syndrome type I. Nature Genet 2000; $24: 71$ - 74

5 Sinclair R, De Berker D. Hereditary and congenital alopecia and hypotrichosis. In: R Dawber (ed). Diseases of Hair and Scalp. Oxford: Blackwell Science, 1997: 151-238

6 Sugio Y, Kajii T. Ruvalcaba syndrome: autosomal dominant inheritance. Am J Med Genet 1984; 19: 741 - 753 